\title{
Genetic Quality Affects the Rate of Male and Female Reproductive Aging Differently in Drosophila melanogaster
}

\author{
Martin Brengdahl, Christopher M. Kimber, Jack Maguire-Baxter, Antonino Malacrinò, \\ and Urban Friberg*
}

IFM Biology, AVIAN Behavioural Genomics and Physiology Group, Linköping University, 58183 Linköping, Sweden

Submitted March 9, 2018; Accepted July 23, 2018; Electronically published October 4, 2018

Dryad data: https://dx.doi.org/10.5061/dryad.5089j74.

\begin{abstract}
AвSTRACт: Males and females often maximize fitness by pursuing different reproductive strategies, with males commonly assumed to benefit more from increased resource allocation into current reproduction. Such investment should trade off with somatic maintenance and may explain why males frequently live shorter than females. It also predicts that males should experience faster reproductive aging. Here we investigate whether reproductive aging and life span respond to condition differently in male and female Drosophila melanogaster, as predicted if sexual selection has shaped male and female resourceallocation patterns. We manipulate condition through genetic quality by comparing individuals inbred or outbred for a major autosome While genetic quality had a similar effect on condition in both sexes, condition had a much larger general effect on male reproductive output than on female reproductive output, as expected when sexual selection on vigor acts more strongly on males. We find no differences in reproductive aging between the sexes in low condition, but in high condition reproductive aging is relatively faster in males. No corresponding sex-specific change was found for life span. The sex difference in reproductive aging appearing in high condition was specifically due to a decreased aging rate in females rather than any change in males. Our results suggest that females age slower than males in high condition primarily because sexual selection has favored sex differences in resource allocation under high condition, with females allocating relatively more toward somatic maintenance than males.
\end{abstract}

Keywords: aging, condition, Drosophila melanogaster, genetic quality, sex differences, sexual selection.

\section{Introduction}

The simple fact that the sexes produce gametes of different sizes (anisogamy) has extensive consequences and may even dictate differences in how males and females age. Because

* Corresponding author; email: urban.friberg@liu.se.

ORCIDs: Brengdahl, http://orcid.org/0000-0002-1052-7274; Kimber, http:// orcid.org/0000-0003-4620-0166; Malacrinò, http://orcid.org/0000-0002-0811-1229; Friberg, http://orcid.org/0000-0001-6112-9586.

Am. Nat. 2018. Vol. 192, pp. 761-772. (C) 2018 by The University of Chicago. 0003-0147/2018/19206-58321\$15.00. All rights reserved.

DOI: $10.1086 / 700117$ males produce vastly more sperm than females produce eggs, each male has the capacity to father many more offspring than any single female can produce (Bateman 1948). This results in male fitness being limited predominately by fertilization opportunities and causes males to compete intensely over access to females (Trivers 1972). Hence, females rarely experience a shortage of potential mates but are instead limited primarily by resources for production of eggs and offspring (Bateman 1948). Anisogamy therefore often results in selection for males and females to employ different reproductive strategies to maximize fitness, which sets the stage for evolution of sex differences in a range of traits, including life histories (Schärer et al. 2012).

One life-history trait that is often observed to differ between the sexes is life span (Promislow 2003; Bonduriansky et al. 2008; Maklakov and Lummaa 2013; Austad and Fischer 2016). In general, it is females that outlive males (Comfort 1979; Trivers 1985; Finch 1990; Promislow 1992), but the opposite also occurs (Promislow et al. 1992; Liker and Székely 2005). Sexual selection is thought to be the main driver behind this pattern (Bonduriansky et al. 2008; Maklakov and Lummaa 2013), although alternative explanations exist (Trivers 1985; Frank and Hurst 1996; Camus et al. 2012). Sexual selection is primarily thought to cause sex differences in life span because in many species males benefit from engaging in activities aimed at attaining access to females that are also associated with risk of injury or death (e.g., territorial fights or display behaviors that attract predators; Williams 1957; Bonduriansky et al. 2008). However, sex differences in life span could also result from sex-specific selection on allocation of limited resources into current reproduction and somatic maintenance. This trade-off has also been suggested to explain much of the variation in aging that we observe in nature (Kirkwood 1977; Kirkwood and Rose 1991; Lemaître et al. 2015), and sexual selection could therefore cause sex differences not only in life span but also in actuarial and reproductive aging (Promislow 2003; Bonduriansky et al. 2008; Maklakov and Lummaa 2013). 
Trivers (1972) offered a conceptual model to explain why sexual selection should often cause sex differences in mortality, and this model can be extended to address sex differences in aging. The model concerns how investment in reproductive effort relates to both current reproductive success and the diminution of future reproductive success, where allocation into reproductive effort is optimized when the net gain in reproductive success is maximized. Trivers casts the diminution of future reproductive success in terms of mortality, but since resources invested in reproductive effort will dictate those remaining for somatic maintenance, the cost can equally be thought of in terms of elevated actuarial or reproductive aging. Trivers's point is that males should generally show higher investment into reproductive effort than females and thus experience shorter life span/ faster aging. This is because male reproductive success is highly dependent on the reproductive effort of other males and because males have the potential for very high reproductive success (but see Kokko and Jennions 2008 for a more general discussion of potential outcomes). Males thus compete over a large potential reward, which requires a high investment in reproductive effort to get a share of (see also Vinogradov 1998).

Notably, Trivers's prediction that sexual selection should result in shorter male life span and by extension faster aging can be reversed. If males accumulate resources or experience with age, which can be translated into high reproductive success only later in life, sexual selection should favor slower male aging than female aging (Graves 2007; Bonduriansky et al. 2008). A combination of the two scenarios is also possible, where males need to accumulate resources over time and enter the mating pool later than females, after which they rapidly invest their resources in competition over females and become the faster-aging sex. Empirical studies that have compared male and female rates of aging mostly show faster male rates, but examples of the opposite also exist (Mysterud et al. 2001; Clutton-Brock and Isvaran 2007; Toïgo et al. 2007; Reed et al. 2008; Nussey et al. 2009; Zajitschek et al. 2009; Dugdale et al. 2011; Archer et al. 2012; Lehtovaara et al. 2013; Beirne et al. 2015; Hayward et al. 2015).

If sexual selection on allocation of resources is a major driver of differences in male and female rates of aging, male and female aging should probably react differently when resource availability is varied (Kokko 2001; Hooper et al. 2018). Predicting which sex will change more when resources are varied over a certain range is difficult, however, without detailed information on the shape of the (supposedly nonlinear) functions that relate reproductive effort to current reproductive success and diminution of future reproductive success in each sex. Resource availability can be manipulated through individual condition, defined as the pool of resources that an individual has accessible for investment into growth, reproductive effort, and somatic maintenance (Rowe and Houle 1996; Hill 2011). A number of studies have indeed shown that condition influences aging. Most of these have focused on only one sex (Bérubé et al. 1999; Bonduriansky and Brassil 2005; Nussey et al. 2006, 2007; Judge et al. 2008; Lee et al. 2008; Adler et al. 2016; Hooper et al. 2017), but a few, limited to two species of crickets, have looked at both sexes simultaneously (Hunt et al. 2004; Maklakov et al. 2009; Zajitschek et al. 2009, 2012; Houslay et al. 2015). In all these studies, variation in condition has primarily been varied through the environmental quality experienced by individuals. Environmental quality during the juvenile and adult stages is an important source of variation in condition, but since male and female condition may be optimized under different environmental conditions (e.g., nutrient intake; Lee et al. 2008; Maklakov et al. 2008), interpreting sex-specific changes in aging after nutrient manipulation may be complicated. Condition is also a function of genetic quality (Rowe and Houle 1996; Hill 2011; Bonduriansky et al. 2015), but rates of aging as a function of genetic quality are little explored. While some small parts of the genome may be sex limited in effect, manipulations of genetic quality that span a large part of the genome and that are averaged across numerous genotypes should render the reduction in condition effectively the same in each sex.

To address whether differences in male and female rates of reproductive aging and life span plausibly result from sexual selection in Drosophila melanogaster, we measured the effect of genetic quality on these traits. Drosophila melanogaster males do not invest in any exaggerated ornaments but compete intensely over matings and allocate substantial time and effort to locating and vigorously courting females. Drosophila melanogaster therefore serves as a model for species under sexual selection. We induce variation in genetic quality by generating matched autosomal inbred and outbred lines. Inbreeding is an excellent way to manipulate condition through genetic quality (Cotton et al. 2004; Tomkins et al. 2004; Bellamy et al. 2014), since the expression of many recessive, mildly deleterious mutations will collectively contribute to reducing an organism's ability to sequester resources throughout all life stages. By exclusively inbreeding an autosome, we avoid potential sex-specific effects generated by inbreeding the $\mathrm{X}$ chromosome only in females. We show that inbreeding reduces condition similarly in the sexes, as measured through reduced egg-to-adult viability, and that male reproductive output is more sensitive to alteration of condition than that of females. Condition had different effects on male and female reproductive aging, with high-condition females aging slower than low-condition females, while reproductive aging was the same in highand low-condition males. Life span declined with low condition but to a similar degree in each sex. Collectively, our results are consistent with the hypothesis that sexual selection 
is an important factor shaping sex differences in reproductive aging in D. melanogaster.

\section{Material and Methods \\ Study Population}

Our experiments used flies from a laboratory-adapted population of Drosophila melanogaster known as Dahomey, named after the location in West Africa (now Benin) where it was collected in 1970. Since that point it has been maintained in the laboratory as a large outbred population, housed in population cages with overlapping generations and under constant conditions ( $12 \mathrm{~L}$ : $12 \mathrm{D}$ light cycle, $25^{\circ} \mathrm{C}, 60 \%$ relative humidity, and a standard yeast/sugar-based food medium), which we maintained during our experiments. The overlapping generation protocol used in Dahomey makes it well suited to the study of reproductive aging because selection is free to act on reproduction throughout life, rather than being confined to a narrow early-life window as typically occurs in lab populations reared on a discrete generation cycle. All data collected within this study have been deposited in the Dryad Digital Repository: https://doi.org/10.5061/dryad.5089j74 (Brengdahl et al. 2018b).

\section{Creation of Chromosome Homozygote Lines}

The genome of $D$. melanogaster is made up of the sex chromosomes (X and $\mathrm{Y}$ ), the two major autosomes (A2 and A3), and the "dot" autosome (A4), which comprises $<1 \%$ of the coding genome. To manipulate genetic quality and by extension condition, we created inbred lines that were completely homozygous for A2, comprising 35\% of the genome (D. melanogaster genome Release 6$)$. These lines were created using an A2 balancer chromosome, which suppresses recombination with its homologue, in concert with the natural absence of recombination in D. melanogaster males. We used this technique to sample focal A2 chromosomes from the Dahomey population at random, which we then amplified and expressed in the heterozygous (outbred) or homozygous (inbred) state in a genetic background randomly sampled from Dahomey.

Here we briefly describe the creation of our A2 inbred lines; see figure S1B in Brengdahl et al. (2018a) for more detail. We began by randomly sampling 44 males from a recently made replica of the Dahomey population that had a marked A2 balancer (CyO, Duox $\left.{ }^{C y}, c n^{2}, d p y^{l v l}, p r^{l}\right)$ backcrossed into it and maintained in a heterozygous state. These males were mated singly to groups of five virgin females that were heterozygous for the dominant A2 marker $K r^{I f-1}$ in an otherwise variable Dahomey background. From each cross involving a single male we collected sons carrying the $K r^{I f-1}$ marker but not the balancer; these therefore all carried the focal Dahomey A2. We mated these sons in groups to virgin females that were heterozygous for the balancer in a variable Dahomey background. Due to the lack of recombination in male D. melanogaster, the focal A2 does not recombine with the marked homologue, so any offspring from this cross that do not express $\mathrm{Kr}^{I f-1}$ carry the intact focal A2. We collected sons and virgin daughters that all carried both the focal A2 and the balancer and crossed them together to generate males and females that had fixed the focal A2. Ten of these 44 initial A2 inbred lines were sterile or inviable in the homozygous state and were lost. We randomly selected 15 of the remaining lines to use in our experiments and maintained them in four vials of 16 pairs to preserve genetic variation elsewhere in the genome.

\section{Egg-to-Adult Viability Assay}

To confirm that inbreeding of A2 produced the expected reduction in condition due to reduced genetic quality, we tested for a drop in egg-to-adult viability in our inbred lines. Viability acts as a good proxy for condition that is comparable between the sexes, as it is a highly polygenic lifehistory trait with a genetic architecture that is largely shared between the sexes (Chippindale et al. 2001) and is known to decrease when condition is reduced through genetic or environmental quality (Bonduriansky et al. 2015). When measuring egg-to-adult viability, we used inbred females from each line to produce both inbred and outbred eggs to avoid conflating the direct genetic effect of inbreeding on condition with any maternal effects. Consequently, we did not control for paternal effects, which do exist in D. melanogaster (e.g., Friberg et al. 2011) but are considered as less important in general. To standardize any environmental impact, we collected these females as virgins after two generations of culture at a controlled density of 120 eggs per vial.

When producing eggs for the viability measurement from each line, 50 virgin females were combined with 25 males from the same line to generate inbred offspring, while another 50 virgin females were combined with 25 randomly selected Dahomey males to generate outbred offspring (fig. 1). Each group mated for 5 days in the presence of ad lib. live yeast to stimulate oviposition. We collected experimental eggs in four blocks on successive days; the eggs collected in each block were all laid in a $2-3$-h window on a small petri dish of food medium. For each line, we separately collected 90 inbred and 90 outbred eggs per block, and each group was placed in a separate vial along with 90 eggs from a competitor population homozygous for a recessive phenotypic marker conferring dark body coloration (ebony, introgressed into the Dahomey background). Egg-to-adult viability was scored 12 days after oviposition by counting all successfully eclosed focal and competitor adults. 


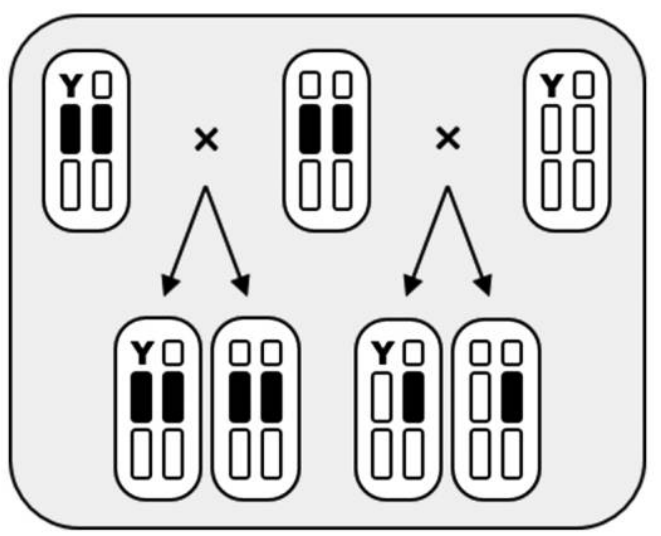

Figure 1: Crossing design to produce matched inbred and outbred genotypes for the autosome A2. Large white rectangles represent individuals/their genotype, and the chromosomal complement is displayed within: the sex chromosomes $\mathrm{X}$ and $\mathrm{Y}$ (as a smaller rectangle and letter $\mathrm{Y}$, respectively) and the major autosomes (A2 and A3) are shown, while the "dot" chromosome (A4) is omitted for clarity. Black rectangles are used to represent a focal A2 chromosome, while white rectangles are chromosome copies sampled from the Dahomey base population and vary randomly between individuals. Focal males and females inbred or outbred for A2 (bottom row, left and right pair, respectively) were both produced using the inbred female genotype as a dam, so inbred and outbred flies in each matched pair experienced similar maternal effects. Outbred focal flies were produced by mating the inbred females to random Dahomey males, while inbred flies were produced by mating the inbred females to males carrying the same cloned A2 chromosome copy (top row).

\section{Lifetime Reproductive Output Assay}

To assess how genetic quality and by extension condition influences adult life span and patterns of reproduction with age in males and females, we simultaneously measured mortality and reproductive output in a competitive environment. For each line we measured the life span of 30 inbred and 30 outbred individuals of each sex, and the same individuals were used to measure reproductive output over their lifetimes. Experimental individuals were produced using a crossing scheme similar to the egg-to-adult viability assay (fig. 1). In this instance, 80 virgin females from each line were mated to 80 males from the same line across four vials to generate experimental inbred males and females, while another $80 \mathrm{vir}$ gin females were mated to 80 random Dahomey males in parallel to generate matched outbred males and females. After females oviposited, all adults were discarded and egg densities standardized to 120 eggs per vial. We used a lower number of eggs here to reduce competition over resources among larvae; using a less resource-limited environment limits any relative phenotypic benefit that inbred larvae could accrue from decreased competition due to reduced viability in in- bred lines. If they do accrue any benefit, it would make our estimates of the impact of inbreeding conservative.

We collected experimental flies 10 days after oviposition under light $\mathrm{CO}_{2}$ anesthesia and split the 30 flies per line, sex, and inbreeding treatment (genetic quality) into two vials of 15 . Two days later, we added 15 same-sex and 30 oppositesex competitors from the ebony-marked population described above to each vial. These competitors were collected 11 days after oviposition and were replaced weekly with a new set of 11-day-old flies under light $\mathrm{CO}_{2}$ anesthesia. We applied this protocol since flies in a stable overlapping-generation population will not simply cohabit with same-aged flies throughout life. Measures of life span and age-specific reproductive output using competitors of a standardized age was thus done in a social context that closely matched the one that flies in the base population normally experience.

All experimental flies were transferred to fresh vials four times a week. At each transfer we scored mortality and removed dead flies. Transfers were done without anesthesia except when we replaced competitors. Once a week we saved the vials between two transfers ( $\sim 18 \mathrm{~h}$ apart). Offspring emerging from these vials were used to assess reproductive output of males and females at that age. Since egg densities varied between vials and ages, we manually adjusted the number of eggs per vial downward after laying to limit overcrowding when necessary. After 12 days, we counted the number of wild-type and ebony flies that had eclosed in these vials. Since ebony is a recessive marker, offspring fathered (mothered) by focal males (females) could be distinguished from those produced by competitor males (females). Reproductive output of focal individuals was estimated relative to competitors in the same vial. The first measurement of reproductive output took place on day 4 of the experiment and thereafter occurred every seventh day for 9 weeks.

\section{Statistical Analysis}

All statistical analyses were carried out using the R statistical environment (R Core Team 2017). The effect of genetic quality (GQ) on egg-to-adult viability and therefore condition was modeled using a generalized linear mixed-effect model with a binomial error distribution and logit link function in the package lme4 (Bates et al. 2017) with the syntax

$$
\begin{aligned}
\text { viability } \sim \operatorname{sex} \times \mathrm{GQ} & +(1 \mid \text { line })+(1 \mid \text { line:sex }) \\
& +(1 \mid \text { line:GQ })+(1 \mid \text { line:sex:GQ }),
\end{aligned}
$$

where the response was the proportion of focal adults to marked competitor adults eclosed. Sex and genetic quality, as well as their interaction, were modeled as fixed effects, while line and its interactions with sex and genetic quality were modeled as random effects. Additionally, we tested a 
model including block as a random effect accounting for variation between days that the experiment was replicated over, but this term did not improve the model fit based on Akaike's information criterion and was therefore omitted from the final model. Tests for significance of the main effects and their interactions were carried out using type III Wald $\chi^{2}$ from the package car and function Anova (Fox and Weisberg 2011).

To reduce potential problems associated with selective deaths when interpreting the effect of genetic quality on reproductive output, we restricted our analysis of reproductive output to the first 5 weeks. By week 5 no more than $13 \%$ of focal flies had died in any combination of line and sex (apart from line 1 where $40 \%$ of inbred females had died), while this number was already up to $27 \%$ in week 6 (and $50 \%$ of inbred females from line 1). However, including week 6 in the models of reproductive output did not qualitatively change our conclusions.

To model the degree of inbreeding depression (ID) in reproductive output, we first standardized reproductive output (SRO). This was accomplished by dividing the number of focal offspring by the number of focal parents producing them (15 in most cases but somewhat fewer in later weeks) and then dividing that quotient by the number of competitor offspring per competitor parent, as follows:

$\mathrm{SRO}=\frac{\text { number of adult focal offspring } / \text { number of focal parents }}{\text { number of adult competitor offspring/number of competitor parents }}$

ID was calculated separately for each sex, line, and week of age (using the mean SRO of the two vials in each aforementioned combination) as follows:

$$
\mathrm{ID}=\frac{\text { outbred SRO }- \text { inbred SRO }}{\text { outbred SRO }} .
$$

The effects of sex and age on ID were then modeled using a linear mixed-effect model in the package lme4 (Bates et al. 2015) with the syntax

$$
\mathrm{ID} \sim \operatorname{sex} \times \text { age }+(1 \mid \text { line })+(1 \mid \text { line }: \text { sex })+(1 \mid \text { line:age }),
$$

where sex, age, and their interaction were modeled as fixed effects and line and its interactions were modeled as random effects. Age was treated as a factor rather than a covariate to enable pairwise contrasts between the sexes at each of the five measurement ages. No three-way interaction of line, sex, and age was included in the model since there was no replication at the level of the interaction. Tests for the significance of main effects and interactions were carried out using type III Wald F-tests from the package car and function Anova (Fox and Weisberg 2011). To perform multiple comparisons of means, we used the package lsmeans and function lsmeans, with pairwise contrasts and the Kenward-Roger approximation of degrees of freedom (Lenth 2016).
To test whether GQ and sex affected the rate of reproductive aging, we used a generalized linear mixed-effect model with a Poisson error distribution and log link function fit using Bayesian Hamiltonian Markov chain Monte Carlo in the package rstanarm (Gabry and Goodrich 2018). We used weakly informative, normally distributed priors for both the intercept (mean $=0, S D=10$ ) and the coefficients (mean $=0, \mathrm{SD}=2.5$ ), with autoscaling for the coefficient priors. We ran four chains with 2,000 iterations each and discarded the first 1,000 as warm-up, after which chain convergence was evaluated using the Gelman-Rubin potential scale reduction factor. This model had the syntax

$$
\begin{aligned}
& \text { reproductive output } \sim \text { sex } \times \text { GQ } \times \text { age }+ \text { offset }(\text { focal }) \\
& + \text { offset }(\text { competitor })+(1 \mid \text { line })+(1 \mid \text { vial }) \\
& +(1 \mid \text { line }: \text { sex })+(1 \mid \text { line }: \text { GQ })+(1 \mid \text { line }: \text { age }) \\
& +(1 \mid \text { line }: \text { sex:GQ })+(1 \mid \text { line }: \text { sex }: \text { age }) \\
& +(1 \mid \text { line: }: G Q: \text { age })+(1 \mid \text { line }: \text { sex }: G Q: \text { age })
\end{aligned}
$$

where the response was the number of adult focal offspring. Sex, GQ, and age, as well as their interactions, were treated as fixed effects (where age is a covariate). Line and its interactions were modeled as random effects. Vial was modeled as a random effect nested within sex, treatment, and line. Offset terms were included to standardize the count data by the log-transformed number of focal parents and the logtransformed offspring-parent competitor ratio. Posterior distributions of the rate of reproductive aging were calculated for each sex/GQ group from the relevant posterior distributions of the model coefficients. The presence of reproductive aging was determined by testing the posterior distribution of the rate of reproductive aging against zero, while comparisons of reproductive aging between groups were made by first calculating the posterior distribution of the difference between the relevant rates and then testing that difference against zero. We report the equivalent to a two-tailed $P$ value for these tests, calculated as two times the proportion of samples in a posterior distribution that was smaller/ greater than zero, whichever was smaller.

Life span was analyzed using a mixed-effect Cox proportional hazard model in the package coxme (Therneau 2018) with the syntax

$$
\text { life span } \sim \operatorname{sex} \times \mathrm{GQ}+(1 \mid \text { line })+(1 \mid \text { vial }),
$$

where sex, GQ, and their interaction were modeled as fixed effects and line and vial were modeled as random effects, with vial nested within sex, genetic quality, and line. We tested for significance of main effects and interactions using type III Wald $\chi^{2}$ from the package car and function Anova (Fox and Weisberg 2011). 


\section{Results \\ Effect of Inbreeding on Viability}

Inbreeding (genetic quality) had a significant negative effect on egg-to-adult viability, while sex did not influence it (genetic quality: $\chi^{2}=7.27, \mathrm{df}=1, P=.007$; sex: $\chi^{2}=$ $0.265, \mathrm{df}=1, P=.607$; eq. [1]), with the reduced viability suffered by inbred individuals indicating that they are indeed in lower condition. The decline (grand mean $\pm \mathrm{SE}$ ) was $8.2 \% \pm 2.7 \%$ for males and $6.8 \% \pm 2.8 \%$ for females. There was no significant interaction between sex and genetic quality, indicating that inbreeding influenced viability of males and females similarly $\left(\chi^{2}=0.138, \mathrm{df}=1, P=.710\right.$; eq. [1]). These results show that the reduction in genetic quality through inbreeding produced a similar decrease in viability and by extension condition in both sexes.

\section{Inbreeding Depression of Reproductive Output}

There was a significant interaction between the impact of sex and age on ID of reproductive output $\left(F_{4,56.0}=6.95\right.$, $P<.001$; eq. [4]; fig. 2), indicating that the difference in ID between the sexes changed with age. The main effects of sex and age both significantly impacted ID as well (sex: $F_{1,69.9}=12.6, \quad P<.001$; week: $F_{4,112}=5.83, \quad P<.001$; eq. [4]; fig. 2). Pairwise contrasts for each week of age revealed significant differences in ID between the sexes for week $1(t=-3.55$, df $=69.9, P<.001)$, week $2(t=$ $-2.43, \mathrm{df}=69.9, P=.018)$, and week $4(t=3.02$, $\mathrm{df}=69.9, P=.004)$ but not for week $3(t=-1.49$, $\mathrm{df}=69.9, P=.141)$ and week $5(t=0.62$, df $=69.9$, $P=.536)$. Female reproductive output showed no ID in

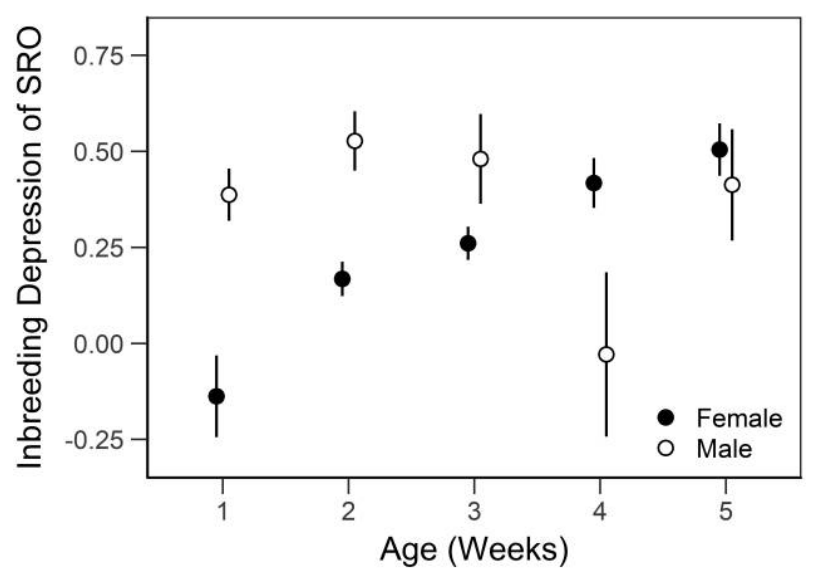

Figure 2: Inbreeding depression of standardized reproductive output (SRO) in males and females for the first 5 weeks of adult life. Circles show the grand mean of individual line means, and error bars represent the SE of the mean. Open circles represent female ID, whereas closed circles represent male ID. week 1, but ID gradually increased over subsequent weeks (fig. 2). Male reproductive output showed ID from week 1 onward, with the exception of week 4 (fig. 2). For an unknown reason, the estimate of male ID for week 4 showed a large departure from the other weeks and was also associated with a much larger error than for the other weeks (see fig. 2). We therefore excluded week 4 data in subsequent analyses for both sexes (but note that including the week 4 data in the subsequent analysis yielded qualitatively similar results).

\section{Effect of Genetic Quality on Male and Female Reproductive Aging}

After confirming that significant reproductive aging occurred in all four sex/GQ groups (all $P<.001$; eq. [5]), we explicitly tested for (i) different rates of reproductive aging between the sexes separated by high and low GQ and (ii) different rates of reproductive aging due to GQ separately in males and females. In high GQ, the rate of reproductive aging differed between the sexes, with females aging slower than males $(P<.001$; eq. [5]), whereas in low GQ the rate of aging did not differ between the sexes $(P=$ .212; eq. [5]). In females, GQ had a significant effect on the rate of aging, with flies in high GQ aging slower than low-GQ flies $(P<.001$; eq. [5]). In males, GQ had no effect on the rate of aging ( $P=.377$; eq. [5]). All results from this section are illustrated in figure 3.

\section{Effect of Genetic Quality on Male and Female Life Span}

Life span was dependent on both sex $\left(\chi^{2}=73.2\right.$, $\mathrm{df}=1$, $P<.001$; eq. [6] $)$ and GQ $\left(\chi^{2}=29.4\right.$, df $=1, P<.001$; eq. [6]), with males living longer than females and high-GQ flies living longer than low-GQ flies (fig. 4). There was, however, no significant interaction between sex and GQ $\left(\chi^{2}=\right.$ 0.510 , df $=1, P=.475$; eq. [6]), indicating that reduced GQ had a similarly negative effect on both sexes.

\section{Discussion}

Males and females are often selected to employ different reproductive strategies to maximize fitness, and these have been suggested to involve sex-specific allocation of available resources into reproduction and somatic maintenance (e.g., Trivers 1972; Bonduriansky et al. 2008). Since investment into somatic maintenance is predicted to influence an individual's future reproduction and survival (Kirkwood and Holliday 1979), sexual selection could hence generate sex differences in life span and reproductive aging. Here we test this hypothesis by varying condition, and thus the pool of accessible resources, through genetic quality. Our manipulation of genetic quality had a similar effect on condition in 

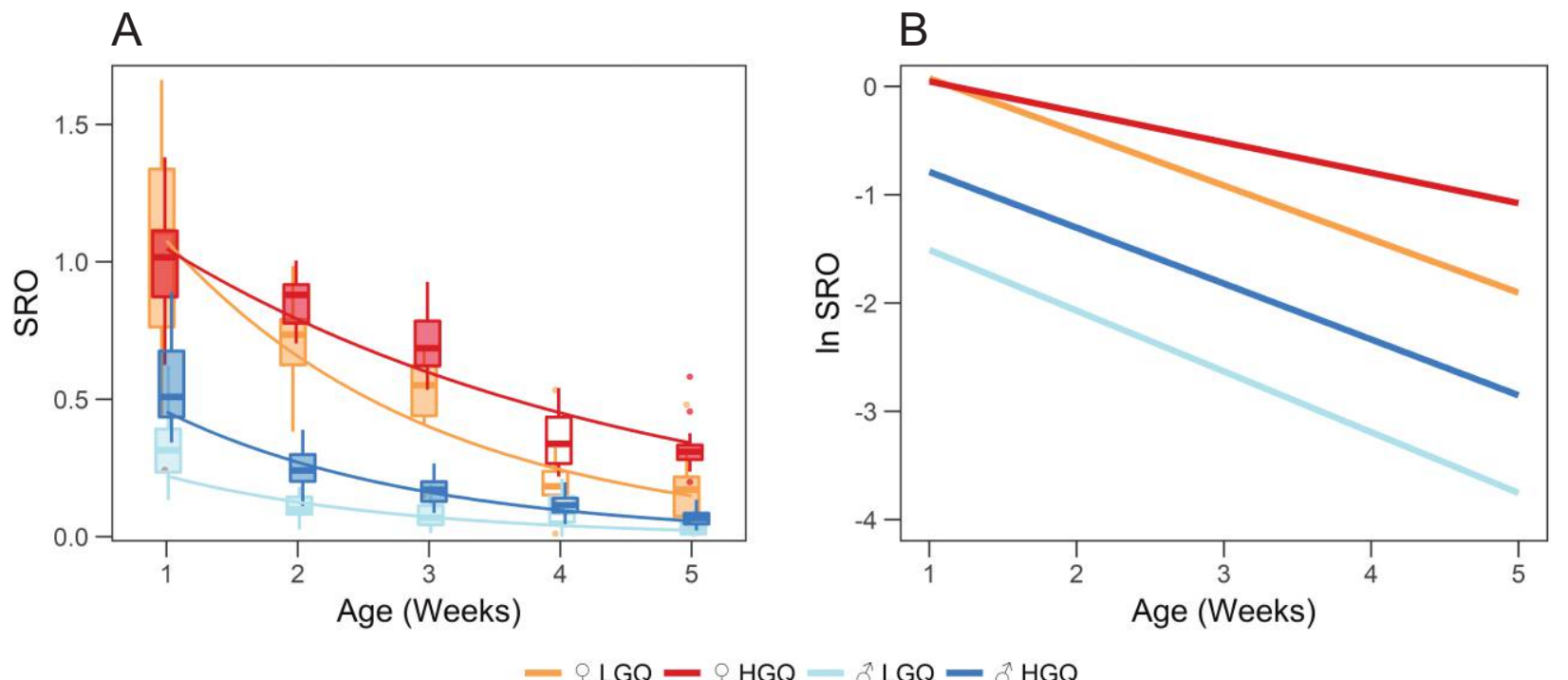

Figure 3: $A$, Standardized reproductive output (SRO) of males and females of low genetic quality (LGQ) and high genetic quality (HGQ), where a score of 1 indicates reproductive performance equal to standardized competitors. The horizontal lines in the boxplots represent medians, the boxes themselves encompass 25 th to 75 th percentiles, and whiskers extend up to 1.5 times the interquartile range. Boxplots of SRO from week 4 (which was excluded from the primary analysis) are rendered without colored fill. Regression lines are derived from the parameter estimates from generalized linear mixed models of reproductive output. $B$, Log-transformed regression lines (from $A$ ) to visualize differences in the rate of reproductive aging between groups on a linear scale.

both sexes, as measured through egg-to-adult viability. Condition in its turn had a large influence on male reproductive output but did not change their rate of reproductive aging. In contrast, condition had a smaller general effect on reproductive output in females, but high-condition females aged slower than both low-condition females and males in either condition. These sex-specific responses of reproductive aging to condition are consistent with sex differences in reproductive aging being generated by sexual selection. The sex-specific change in reproductive aging was not mirrored for life span, for which low-condition individuals of both sexes showed a similar reduction. Below we discuss our results in more detail.

In our experiment, we replaced male and female competitor flies on a weekly basis. This mimics conditions in populations with overlapping generations such as the one we study, where flies are exposed to a constant demographic structure throughout life. Replacement also made it possible to take a standardized weekly measure of reproductive output, from which we could estimate the rate of reproductive aging. Although replacement of competitor flies was identical for both sexes, males produced fewer offspring than did females on average, something that is not possible in sexually reproducing species under natural conditions. The reason behind this occurrence in our experiment is probably because during the replacement of competitor females housed with focal males the new females added had already mated with competitor males. This effectively gave the competitor males a head start in the competition over fertilization opportunities and likely explains the higher fraction of offspring sired by competitor males even though both competitor and focal males could subsequently compete for access to females on equal footing. Focal females, in contrast, had already mated when competitor females were replaced, leaving focal and competitor females on equal footing throughout. Our fre-

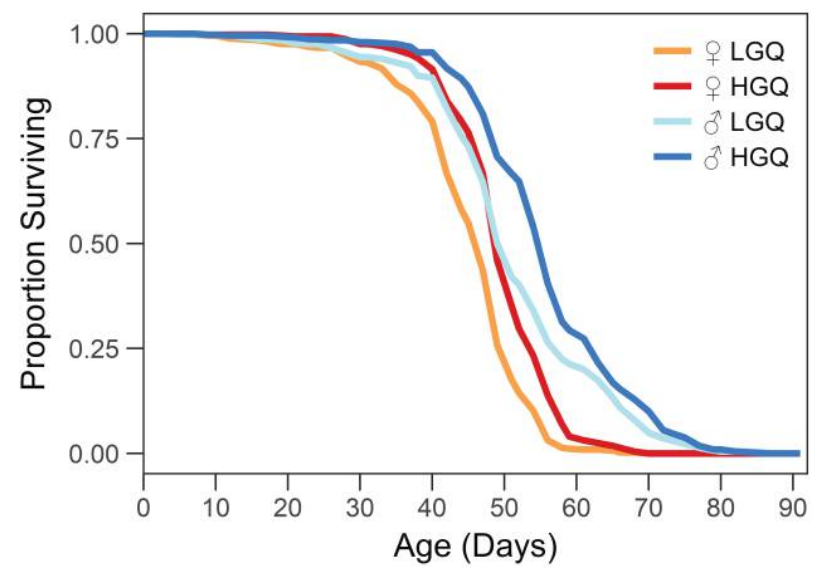

Figure 4: Proportion of male and female flies of low genetic quality (LGQ) and high genetic quality (HGQ) surviving to a given day of age. For each sex and genetic quality combination, the survival of 450 focal flies was followed in total, summing over the 15 lines used in the assay. 
quent replacement of competitor flies also likely explains why females had a shorter average life span than males in our experiments. In previous studies of our study population, we and others (e.g., Magwere et al. 2004; Lehtovaara et al.2013; Griffin et al. 2016; Duxbury et al. 2017) have found that females outlive males. The degree of exposure to males does, however, have a large impact on survival of Drosophila melanogaster females (Partridge et al. 1987; Friberg 2005; Lehtovaara et al. 2013; Zajitschek et al. 2013), and the fact that we replaced competitors more frequently than in any of the aforementioned studies therefore probably caused the shorter female life span.

To be able to directly compare how altering condition changes the rate of aging in males versus females, it is important that condition itself is altered to the same extent in both sexes. When we compared male and female reproductive output in early life, when the majority of reproduction takes place, the reduction in relative reproductive output between high- and low-condition treatments was considerably larger in males than in females. This may at first appear as if our manipulation of genetic quality had a larger influence on condition in males. Instead, we attribute this larger difference in males to them experiencing stronger sexual selection-a compelling argument, as this effect is believed to be behind the stronger selection against deleterious mutations commonly observed in males compared to females (Whitlock and Agrawal 2009; Mallet and Chippindale 2011; Mallet et al. 2011; Sharp and Agrawal 2013; Grieshop et al. 2016; but see Allen et al. 2017; Prokop et al. 2017). An alternative explanation for this pattern is that $D$. melanogaster males have more sex-limited transcripts than do females overall, as the testes are particularly enriched for sex-limited gene expression (Graveley et al. 2011). Inbred males may thus express more deleterious mutations than females, but given that their effect should primarily be limited to the function of the testis and male ejaculate, they probably have a negligible effect on male condition. When we instead measure how genetic quality influences condition through egg-to-adult viability, a trait for which males and females have a common genetic architecture (Chippindale et al. 2001; Mallet and Chippindale 2011) and on which sexual selection does not act, we see a similar effect on both males and females. Given that our manipulation of genetic quality had a similar effect on condition in both sexes, we can thus compare how condition changes male and female reproductive aging on the same scale.

Males showed faster reproductive aging than females in high condition. This result is consistent with a scenario where males invest more into reproductive effort and less into somatic maintenance compared to females. If this was the case, males should also show faster physiological deterioration than females. The pace of whole-organism physiological deterioration is difficult to assess, but one promising index for flies is the speed with which they climb up culture vials when disturbed (Gargano et al. 2005). We did not undertake such an assay here, but note that males lived longer than females in our experiments, which speaks against faster male physiological deterioration than female physiological deterioration. As discussed above, however, the fact that females were constantly exposed to harassment from young vigorous males probably generated large sex differences in mortality rate, especially when flies became old and fragile. This may thus have reversed the expected relationship in life span based on intrinsic physiological deterioration.

Faster reproductive aging in males than in females can also occur in the absence of faster male physiological deterioration, if physiological status (which generally declines with age) has a steeper relationship with reproductive success in males compared to females (Nussey et al. 2009). In other words, male reproductive success could be more sensitive to the same unit of physiological change than female reproductive success. This is expected to occur when competition between males over access to females results in stronger overall selection on male vigor than female vigor. Our results suggest that this pattern of selection, as discussed above, and the faster male reproductive aging we observe in highcondition individuals could therefore be at least partly explained by this mechanism. Faster male reproductive aging due to faster physiological deterioration or faster exclusion from the mating pool are both compatible with a scenario where sex differences in aging are caused by sexual selection but differ in the sense that the former influences the phenotypes of males and females while the latter merely alters male and female reproductive schedules differently according to condition. These two hypotheses are not mutually exclusive, and further experiments are needed to evaluate their relative contribution to faster male reproductive aging.

In contrast to the difference in reproductive aging for males and females in high condition, no sex difference in reproductive aging was observed in low condition. This change in the relative rate of aging between the sexes was caused by faster reproductive aging of low-condition females, while lowcondition males displayed the same rate of reproductive aging as high-condition males. These results may indicate that lowcondition males have nothing to gain from investing relatively less than high-condition males into reproductive effort, because this strategy would effectively exclude them from the mating pool at all times. High-condition females may on the other hand gain more from increased investment into somatic maintenance, since female fitness is primarily resource limited. Allocating an extra energy unit into reducing reproductive aging may therefore pay off more than investing it into current reproduction, which is also supported by some theory (e.g., Ellers et al. 2000; Rosenheim et al. 2008; Berger et al. 2012). The fact that reproductive aging reacts differently in males and females when condition is manipulated is consistent with the idea that males and females trade off resources 
differently and that this difference in turn results from sexual selection on allocation patterns in the evolutionary past.

In contrast to our finding that reproductive aging changed differently with condition in males and females, our manipulation of condition had an almost identical effect on male and female life span. A possible explanation is that reproduction and life span are at least partly disconnected (reviewed in Partridge et al. 2005). While the general evolutionary theory of aging suggests that all traits should age in concert, several studies comparing the rate of aging of different phenotypes have concluded that this is not necessarily the case (Nussey et al. 2009; Cornwallis et al. 2014). It is conceivable that the rates of reproductive and actuarial aging respond more similarly to condition in each sex despite the mismatch between reproductive aging and life span; our study was not designed to directly estimate actuarial aging at the line level. Despite finding results consistent with sexual selection shaping sex differences in resource allocation to either reproduction or somatic maintenance, we thus find no evidence that this in turn drives sex differences in life span.

Since we did not directly manipulate the strength of sexual selection in these experiments, we can only infer that the sex differences in reproductive aging we observe between high- and low-condition individuals are the result of sexual selection. Apart from sexual selection, asymmetrical inheritance of cytoplasmic genomes (Frank and Hurst 1996; Gemmell et al. 2004) and unconditional expression of recessive deleterious mutations in the heterogametic sex (the unguarded-X hypothesis; Trivers 1985) have also been suggested as drivers of sex differences in life span and by extension aging (Bonduriansky et al. 2008). There is support for mitochondria playing a role in aging (e.g., Maklakov et al. 2006; Dowling et al. 2009) and also for mutations in the mitochondrial genome having male-biased deleterious effects on life span (Camus et al. 2012). However, it is difficult to imagine how these effects could mediate female-specific responses in reproductive aging when condition is altered, especially here where we controlled for maternal effects. No support for the unguarded-X hypothesis was found in the most direct test of this hypothesis yet (Brengdahl et al. $2018 a$ ), but more indirect tests have found patterns consistent with this possibility (Pipoly et al. 2015; Carazo et al. 2016; Sultanova et al. 2018). In any case, it also seems difficult to construct a scenario where $\mathrm{X}$-linked recessive mutations could cause a response in female but not male reproductive aging when condition is altered. We therefore conclude that the most plausible explanation for the sex-specific change in reproductive aging we observe in response to condition is past sexual selection on allocation patterns.

Our method of manipulating genetic quality and condition through inbreeding of an autosome has been used previously in aging research but from a different perspective. The mutation accumulation (MA) theory of aging predicts that aging is caused by late-acting (recessive) deleterious mutations and thus predicts elevated inbreeding depression at late ages (Charlesworth and Hughes 1996). Studies testing this hypothesis by comparing outbred and inbred individuals have in general found support for elevated inbreeding with age in D. melanogaster (e.g., Charlesworth and Hughes 1996; Hughes et al. 2002; Snoke and Promislow 2003; Reynolds et al. 2007). However, using inbreeding depression to evaluate the MA theory of aging ignores that the many recessive deleterious mutations that already have an effect early in life will influence the efficiency with which individuals acquire and metabolize resources (i.e., condition). This will in turn reduce what resources can be invested into somatic maintenance. It is thus likely that the effect of these early-acting deleterious mutations, which have nothing to do with aging under an MA scenario, determines the performance of individuals during late life and obscures the impact of any late-life acting mutations (for a similar argument, see Hughes and Reynolds 2005). The fact that we see a clear difference between the sexes in the response to inbreeding with age in this experiment, with a substantial effect on females and none on males, supports the view that inbreeding has a much larger influence on aging through condition than through expression of late-acting mutations. For the recessive deleterious late-acting mutations predicted by MA to explain this result, such mutations would have to be exclusively female limited in their effects, which seems highly implausible.

In summary, we conclude that sexual selection on resourceallocation patterns has plausibly contributed to sex differences in the rate of reproductive aging in our study population. Our data provide no support for the faster male-to-female aging rate we observe in high-condition individuals being driven by high-condition males investing extra resources primarily into current reproduction but instead suggest that faster male reproductive aging in high condition is explained by females primarily investing extra resources into somatic maintenance.

\section{Acknowledgments}

We thank David Berger, Russell Bonduriansky, Stephen Chenoweth, and one anonymous reviewer for constructive comments on the manuscript. The work was financially supported by Helge Ax:son Johnsons stiftelse to M.B., the Royal Physiographic Society in Lund to C.M.K., and the Swedish Research Council to U.F. A.M. was supported by a fellowship from the Lawski Foundation.

\section{Literature Cited}

Adler, M. I., M. Telford, and R. Bonduriansky. 2016. Phenotypes optimized for early-life reproduction exhibit faster somatic deterioration with age, revealing a latent cost of high condition. Journal of Evolutionary Biology 29:2436-2446. 
Allen, S. L., K. McGuigan, T. Connallon, M. W. Blows, and S. F. Chenoweth. 2017. Sexual selection on spontaneous mutations strengthens the between-sex genetic correlation for fitness. Evolution 71:2398-2409.

Archer, C. R., F. Zajitschek, S. K. Sakaluk, N. J. Royle, and J. Hunt. 2012. Sexual selection affects the evolution of lifespan and ageing in the decorated cricket Gryllodes sigillatus. Evolution 66:620-634.

Austad, S. N., and K. E. Fischer. 2016. Sex differences in lifespan. Cell Metabolism 23:1022-1033.

Bateman, A. J. 1948. Intra-sexual selection in Drosophila. Heredity 2:349-368

Bates, D., M. Mächler, B. Bolker, and S. Walker. 2015. Fitting linear mixed-effects models using lme4. Lournal of Statistical Software 67:1-48.

. 2017. lme4: linear mixed-effects models using Eigen and S4. R package version 1.1-13. https://CRAN.R-project.org/package $=$ lme4

Beirne, C., R. Delahay, and A. Young. 2015. Sex differences in senescence: the role of intra-sexual competition in early adulthood. Proceedings of the Roval Society B 282:20151086.

Bellamy, L., K. Fowler, and A. Pomiankowski. 2014. The use of inbreeding to assess the genetic component of condition underlying GEIs in sexual traits. Pages 213-240 in J. Hunt and D. J. Hosken, eds. Genotype-by-environment interactions and sexual selection. Wiley, Hoboken, NJ.

Berger, D., M. Olofsson, M. Friberg, B. Karlsson, C. Wiklund, and K. Gotthard. 2012. Intraspecific variation in body size and the rate of reproduction in female insects - adaptive allometry or biophysical constraint? Journal of Animal Ecology 81:1244-1258.

Bérubé, C. H., M. Festa-Bianchet, and J. T. Jorgenson. 1999. Individual differences, longevity, and reproductive senescence in bighorn ewes. Ecology 80:2555-2565.

Bonduriansky, R., and C. E. Brassil. 2005. Reproductive ageing and sexual selection on male body size in a wild population of antler flies (Protopiophila litigata). Journal of Evolutionarv Biology 18:13321340.

Bonduriansky, R., A. Maklakov, F. Zajitschek, and R. Brooks. 2008 Sexual selection, sexual conflict and the evolution of ageing and life span. Functional Ecology 22:443-453.

Bonduriansky, R., M. A. Mallet, D. Arbuthnott, V. Pawlowsky-Glahn, J. J. Egozcue, and H. D. Rundle. 2015. Differential effects of genetic vs. environmental quality in Drosophila melanogaster suggest multiple forms of condition dependence. Ecology Letters 18:317-326.

Brengdahl, M., C. M. Kimber, J. Maguire-Baxter, and U. Friberg. 2018a. Sex differences in life span: females homozygous for the $\mathrm{X}$ chromosome do not suffer the shorter life span predicted by the unguarded $\mathrm{X}$ hypothesis. Evolution 72:568-577. doi:10.1111/evo.13434.

Brengdahl, M., C. M. Kimber, J. Maguire-Baxter, A. Malacrinò, and U. Friberg. 2018b. Data from: Genetic quality affects the rate of male and female reproductive aging differently in Drosophila melanogaster. American Naturalist, Dryad Digital Repository, https://doi .org/10.5061/dryad.5089j74.

Camus, F., D. J. Clancy, and D. K. Dowling. 2012. Mitochondria, maternal inheritance, and male aging. Current Biology 22:1717-1721.

Carazo, P., J. Green, I. Sepil, T. Pizzari, and S. Wigby. 2016. Inbreeding removes sex differences in lifespan in a population of Drosophila melanogaster. Biology Letters 12:20160337.

Charlesworth, B., and K. A. Hughes. 1996. Age-specific inbreeding depression and components of genetic variance in relation to the evolution of senescence. Proceedings of the National Academv of Sciences of the USA 93:6140-6145.
Chippindale, A. K., J. R. Gibson, and W. R. Rice. 2001. Negative genetic correlation for adult fitness between sexes reveals ontogenetic conflict in Drosophila. Proceedings of the National Academy of Sciences of the USA 98:1671-1675.

Clutton-Brock, T. H., and K. Isvaran. 2007. Sex differences in ageing in natural populations of vertebrates. Proceedings of the Roval Societv B 274:3097-3104.

Comfort, A. 1979. The biology of senescence. Elsevier, New York.

Cornwallis, C. K., R. Dean, and T. Pizzari. 2014. Sex-specific patterns of aging in sexual ornaments and gametes. American Naturalist 184: E66-E78.

Cotton, S., K. Fowler, and A. Pomiankowski. 2004. Do sexual ornaments demonstrate heightened condition-dependent expression as predicted by the handicap hypothesis? Proceedings of the Roval Society B 271:771-783.

Dowling, D. K., A. A. Maklakov, U. Friberg, and F. Hailer. 2009. Applying the genetic theories of ageing to the cytoplasm: cytoplasmic genetic covariation for fitness and lifespan. Iournal of Evolutionary Biology 22:818-827.

Dugdale, H. L., L. C. Pope, C. Newman, D. W. Macdonald, and T. Burke. 2011. Age-specific breeding success in a wild mammalian population: selection, constraint, restraint and senescence. $\mathrm{Mo}-$ lecular Ecology 20:3261-3274.

Duxbury, E. M. L., W. G. Rostant, and T. Chapman. 2017. Manipulation of feeding regime alters sexual dimorphism for lifespan and reduces sexual conflict in Drosophila melanogaster. Proceedings of the Roval Societv B 284:20170391.

Ellers, J., J. G. Sevenster, and G. Driessen. 2000. Egg load evolution in parasitoids. American Naturalist 156:650-665.

Finch, C. E. 1990. Longevity, senescence, and the genome. University of Chicago Press, Chicago.

Fox, J., and S. Weisberg. 2011. An R companion to applied regression. Sage, Thousand Oaks, CA.

Frank, S. A., and L. D. Hurst. 1996. Mitochondria and male disease. Nature 383:224.

Friberg, U. 2005. Genetic variation in male and female reproductive characters associated with sexual conflict in Drosophila melanogaster. Behavior Genetics 35:455-462.

Friberg, U., A. D. Stewart, and W. R. Rice. 2011. X- and Y-chromosome linked paternal effects on a life-history trait. Biology Letters 8:7173.

Gabry, J., and B. Goodrich. 2018. rstanarm: Bayesian applied regression modeling via Stan. R package version 2.17.4. http://mc-stan .org/rstanarm.

Gargano, J. W., I. Martin, P. Bhandari, and M. S. Grotewiel. 2005. Rapid iterative negative geotaxis (RING): a new method for assessing agerelated locomotor decline in Drosophila. Experimental Gerontology 40:386-395.

Gemmell, N. J., V. J. Metcalf, and F. W. Allendorf. 2004. Mother's curse: the effect of mtDNA on individual fitness and population viability. Trends in Ecology and Evolution 19:238-244.

Graveley, B. R., A. N. Brooks, J. W. Carlson, M. O. Duff, J. M. Landolin, L. Yang, C. G. Artieri, et al. 2011. The developmental transcriptome of Drosophila melanogaster. Nature 471:473-479.

Graves, B. M. 2007. Sexual selection effects on the evolution of senescence. Evolutionary Ecology 21:663-668.

Grieshop, K., J. Stangberg, I. Martinossi-Allibert, G. Arnqvist, and D. Berger. 2016. Strong sexual selection in males against a mutation load that reduces offspring production in seed beetles. Journal of Evolutionary Biology 29:1201-1210. 
Griffin, R. M., H. Schielzeth, and U. Friberg. 2016. Autosomal and Xlinked additive genetic variation for lifespan and aging: comparisons within and between the sexes in Drosophila melanogaster. G3 6:39033911.

Hayward, A. D., J. Moorad, C. E. Regan, C. Berenos, J. G. Pilkington, J. M. Pemberton, and D. H. Nussey. 2015. Asynchrony of senescence among phenotypic traits in a wild mammal population. Experimental Gerontology 71:56-68.

Hill, G. E. 2011. Condition-dependent traits as signals of the functionality of vital cellular processes. Ecology Letters 14:625-634.

Hooper, A. K., J. Lehtonen, L. E. Schwanz, and R. Bonduriansky. 2018. Sexual competition and the evolution of condition-dependent ageing. Evolution Letters 2:37-48.

Hooper, A. K., F. Spagopoulou, Z. Wylde, A. A. Maklakov, and R. Bonduriansky. 2017. Ontogenetic timing as a condition-dependent life history trait: high-condition males develop quickly, peak early and age fast. Evolution 71:671-685.

Houslay, T. M., J. Hunt, M. C. Tinsley, and L. F. Bussiere. 2015. Sex differences in the effects of juvenile and adult diet on age-dependent reproductive effort. Journal of Evolutionary Biology 28:1067-1079.

Hughes, K. A., J. A. Alipaz, J. M. Drnevich, and R. M. Reynolds. 2002. A test of evolutionary theories of senescence. Proceedings of the National Academy of Sciences of the USA 99:14286-14291.

Hughes, K. A., and R. M. Reynolds. 2005. Evolutionary and mechanistic theories of aging. Annual Review of Entomology 50:421-445.

Hunt, J., R. Brooks, M. D. Jennions, M. J. Smith, C. L. Bentsen, and L. F. Bussiere. 2004. High-quality male field crickets invest heavily in sexual display but die young. Nature 432:1024-1027.

Judge, K. A., J. J. Ting, and D. T. Gwynne. 2008. Condition dependence of male life span and calling effort in a field cricket. Evolution 62:868-878.

Kirkwood, T. B. L. 1977. Evolution of aging. Nature 270:301-304.

Kirkwood, T. B. L., and R. R. S. Holliday. 1979. The evolution of ageing and longevity. Proceedings of the Roval Society B 205:531-546.

Kirkwood, T. B. L., and M. R. Rose. 1991. Evolution of senescence: late survival sacrificed for reproduction. Philosophical Transactions of the Roval Society B 332:15-24.

Kokko, H. 2001. Fisherian and "good genes" benefits of mate choice: how (not) to distinguish between them. Ecology Letters 4:322-326.

Kokko, H., and M. D. Jennions. 2008. Parental investment, sexual selection and sex ratios. Journal of Evolutionary Biology 21:919948.

Lee, K. P., S. J. Simpson, F. J. Clissold, R. Brooks, J. W. O. Ballard, P. W. Taylor, N. Soran, and D. Raubenheimer. 2008. Lifespan and reproduction in Drosophila: new insights from nutritional geometry. Proceedings of the National Academy of Sciences of the USA 105 2498-2503.

Lehtovaara, A., H. Schielzeth, I. Flis, and U. Friberg. 2013. Heritability of life span is largely sex-limited in fruit flies. American Naturalist 182:653-665

Lemaître, J.-F., V. Berger, C. Bonenfant, M. Douhard, M. Gamelon, F. Plard, and J.-M. Gaillard. 2015. Early-late life trade-offs and the evolution of ageing in the wild. Proceedings of the Roval Society B 282:20150209.

Lenth, R. V. 2016. Least-squares means: the R package lsmeans. Journal of Statistical Software 69:1-33.

Liker, A., and T. Szekely. 2005. Mortality costs of sexual selection and parental care in natural populations of birds. Evolution 59:890-897.

Magwere, T., T. Chapman, and L. Partridge. 2004. Sex differences in the effect of dietary restriction on life span and mortality rates in female and male Drosophila melanogaster. Journals of Gerontology A 59:3-9.

Maklakov, A. A., U. Friberg, D. K. Dowling, and G. Arnqvist. 2006 Within-population variation in cytoplasmic genes affects female life span and aging in Drosophila melanogaster. Evolution 60:2081-2086.

Maklakov, A. A., M. D. Hall, S. J. Simpson, J. Dessmann, F. J. Clissold, F. Zajitschek, S. P. Lailvaux, D. Raubenheimer, R. Bonduriansky, and R. C. Brooks. 2009. Sex differences in nutrientdependent reproductive ageing. Aging Cell 8:324-330.

Maklakov, A. A., and V. Lummaa. 2013. Evolution of sex differences in lifespan and aging: causes and constraints. BioEssays 35:717-724.

Maklakov, A. A., S. J. Simpson, F. Zajitschek, M. Hall, J. Dessman, F. Clissold, D. Raubenheimer, R. Bonduriansky, and R. C. Brooks. 2008. Sex-specific fitness effects of nutrient intake on reproduction and lifespan. Current Biology 18:1062-1066.

Mallet, M. A., J. M. Bouchard, C. M. Kimber, and A. K. Chippindale. 2011. Experimental mutation-accumulation on the X chromosome of Drosophila melanogaster reveals stronger selection on males than females. BMC Evolutionary Biology 11:156.

Mallet, M. A., and A. K. Chippindale. 2011. Inbreeding reveals stronger net selection on Drosophila melanogaster males: implications for mutation load and the fitness of sexual females. Heredity 106:994-1002.

Mysterud, A., N. G. Yoccoz, N. C. Stenseth, and R. Langvatn. 2001. Effects of age, sex and density on body weight of Norwegian red deer: evidence of density-dependent senescence. Proceedings of the Royal Society B 268:911-919.

Nussey, D. H., L. E. B. Kruuk, A. Donald, M. Fowlie, and T. H. CluttonBrock. 2006. The rate of senescence in maternal performance increases with early-life fecundity in red deer. Ecology Letters 9:13421350 .

Nussey, D. H., L. E. B. Kruuk, A. Morris, M. N. Clements, J. M. Pemberton, and T. H. Clutton-Brock. 2009. Inter- and intrasexual variation in aging patterns across reproductive traits in a wild red deer population. American Naturalist 174:342-357.

Nussey, D. H., L. E. B. Kruuk, A. Morris, and T. H. Clutton-Brock. 2007. Environmental conditions in early life influence ageing rates in a wild population of red deer. Current Biology 17:R1000-R1001

Partridge, L., D. Gems, and D. J. Withers. 2005. Sex and death: what is the connection? Cell 120:461-472.

Partridge, L., A. Green, and K. Fowler. 1987. Effects of egg production and of exposure to males on female survival in Drosophila melanogaster. Journal of Insect Physiology 33:745-749.

Pipoly, I., V. Bokony, M. Kirkpatrick, P. F. Donald, T. Szekely, and A. Liker. 2015. The genetic sex-determination system predicts adult sex ratios in tetrapods. Nature 527:91-94.

Prokop, Z. M., M. A. Prus, T. S. Gaczorek, K. Sychta, J. K. Palka, A. Plesnar-Bielak, and M. Skarbon. 2017. Do males pay for sex? sexspecific selection coefficients suggest not. Evolution 71:650-661.

Promislow, D. 1992. Costs of sexual selection in natural populations of mammals. Proceedings of the Roval Society B 247:203-210.

. 2003. Mate choice, sexual conflict, and evolution of senescence. Behavior Genetics 33:191-201.

Promislow, D. E. L., R. Montgomerie, and T. E. Martin. 1992. Mortality costs of sexual dimorphism in birds. Proceedings of the Roval Society B 250:143-150.

R Development Core Team. 2017. R: a language and environment for statistical computing. R Foundation for Statistical Computing, Vienna. http://www.R-project.org.

Reed, T. E., L. E. B. Kruuk, S. Wanless, M. Frederiksen, E. A. J. Cunningham, and M. P. Harris. 2008. Reproductive senescence 
in a long-lived seabird: rates of decline in late-life performance are associated with varying costs of early reproduction. American Naturalist 171:E89-E101.

Reynolds, R. M., S. Temiyasathit, M. M. Reedy, E. A. Ruedi, J. M. Drnevich, J. Leips, and K. A. Hughes. 2007. Age specificity of inbreeding load in Drosophila melanogaster and implications for the evolution of late-life mortality plateaus. Genetics 177:587-595.

Rosenheim, J. A., S. J. Jepsen, C. E. Matthews, D. S. Smith, and M. R. Rosenheim. 2008. Time limitation, egg limitation, the cost of oviposition and lifetime reproduction by an insect in nature. American Naturalist 172:487-496.

Rowe, L., and D. Houle. 1996. The lek paradox and the capture of genetic variance by condition dependent traits. Proceedings of the Roval Society B 263:1415-1421.

Schärer, L., L. Rowe, and G. Arnqvist. 2012. Anisogamy, chance and the evolution of sex roles. Trends in Ecology and Evolution 27:260 264.

Sharp, N. P., and A. F. Agrawal. 2013. Male-biased fitness effects of spontaneous mutations in Drosophila melanogaster. Evolution 67: 1189-1195.

Snoke, M. S., and D. E. L. Promislow. 2003. Quantitative genetic tests of recent senescence theory: age-specific mortality and male fertility in Drosophila melanogaster. Heredity 91:546-556.

Sultanova, Z., M. Andic, and P. Carazo. 2018. The "unguarded-X" and the genetic architecture of lifespan: inbreeding results in a potentially maladaptive sex-specific reduction of female lifespan in Drosophila melanogaster. Evolution 72:540-552.

Therneau, T. 2018. coxme. R package version 2.2-7. https://CRAN.R -project.org $/$ package $=$ coxme.

Toïgo, C., J. M. Gaillard, M. Festa-Bianchet, E. Largo, J. Michallet, and D. Maillard. 2007. Sex- and age-specific survival of the highly dimorphic Alpine ibex: evidence for a conservative life-history tactic. Journal of Animal Ecology 76:679-686.

Tomkins, J. L., J. Radwan, J. S. Kotiaho, and T. Tregenza. 2004. Genic capture and resolving the lek paradox. Trends in Ecology and Evolution 19:323-328.

Trivers, R. 1972. Parental investment and sexual selection. Pages 136179 in B. Campbell, ed. Sexual selection and the descent of man. Aldine, Chicago.

. 1985. Social evolution. Benjamin/Cummings, Menlo Park, CA

Vinogradov, A. E. 1998. Male reproductive strategy and decreased longevity. Acta Biotheoretica 46:157-160.

Whitlock, M. C., and A. F. Agrawal. 2009. Purging the genome with sexual selection: reducing mutation load through selection on males. Evolution 63:569-582.

Williams, G. C. 1957. Pleiotropy, natural selection, and the evolution of senescence. Evolution 11:398-411.

Zajitschek, F., J. Hunt, M. D. Jennions, M. D. Hall, and R. C. Brooks, 2009. Effects of juvenile and adult diet on ageing and reproductive effort of male and female black field crickets, Teleogryllus commodus. Functional Ecology 23:602-611.

Zajitschek, F., S. P. Lailvaux, J. Dessmann, and R. C. Brooks. 2012. Diet, sex, and death in field crickets. Ecology and Evolution 2:16271636.

Zajitschek, F., S. R. K. Zajitschek, U. Friberg, and A. A. Maklakov. 2013. Interactive effects of sex, social environment, dietary restriction and methionine on survival and reproduction in fruit flies. AGE 35:1193-1204.

Associate Editor: Stephen F. Chenoweth Editor: Russell Bonduriansky

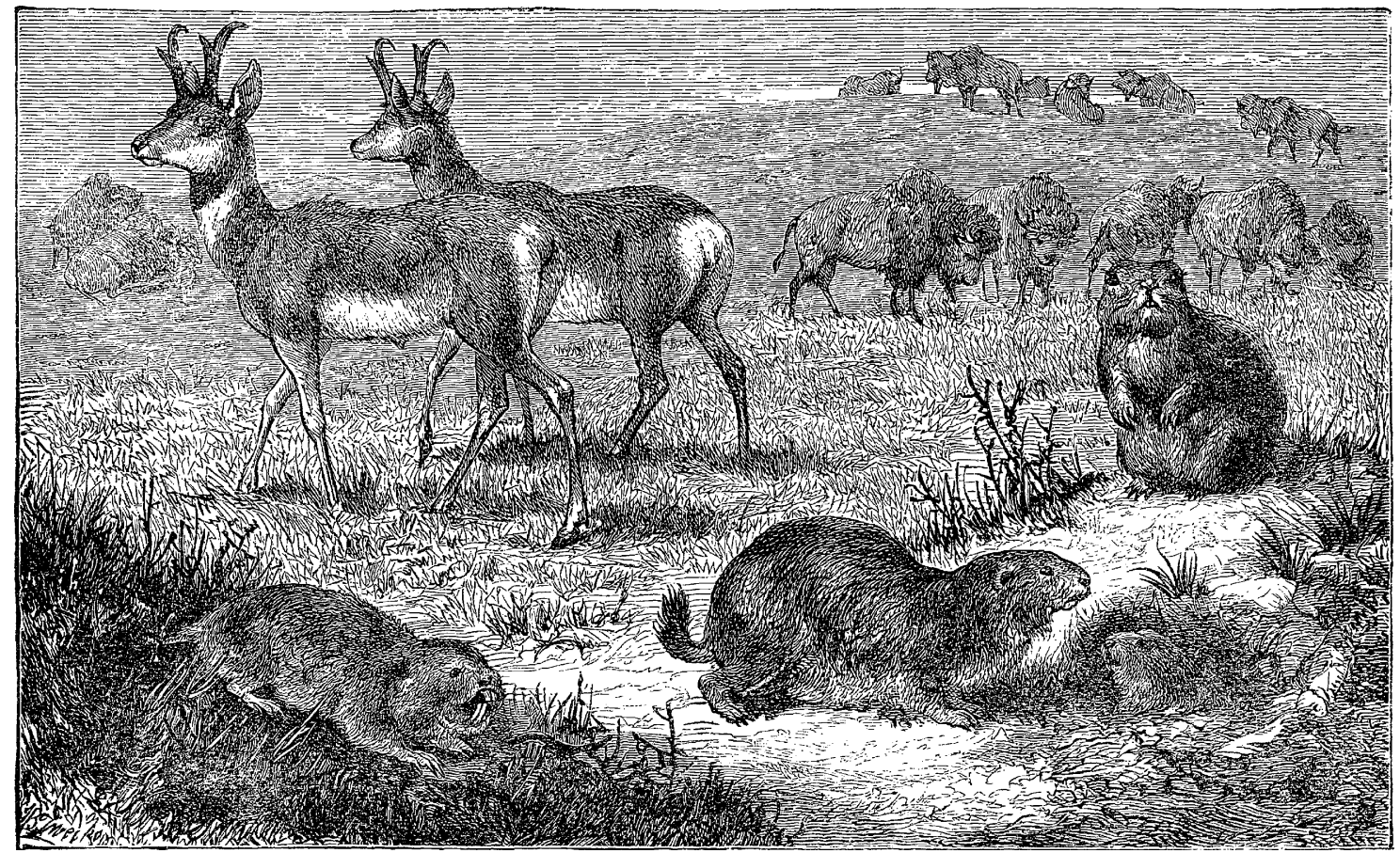

"The North American plains, with characteristic Mammalia." From the review of Wallace's Geographical Distribution of Animals (The American Naturalist, 1877, 11:232-238). 